\title{
Mary Douglas et la Bible
}

La (re)conversion d'une anthropologue

Mary Douglas and the Bible. The (re)Conversion of an Anthropologist

\section{Christophe Lemardelé}

\section{(2) OpenEdition}

\section{Journals}

Édition électronique

URL : http://journals.openedition.org/lhomme/23763

DOI : 10.4000//homme.23763

ISSN : 1953-8103

Éditeur

Éditions de l'EHESS

\section{Édition imprimée}

Date de publication : 27 octobre 2014

Pagination : 139-158

ISSN : 0439-4216

\section{Référence électronique}

Christophe Lemardelé, « Mary Douglas et la Bible », L'Homme [En ligne], 212 | 2014, mis en ligne le 24 octobre 2016, consulté le 19 avril 2019. URL : http://journals.openedition.org/lhomme/23763 ; DOI : 10.4000//homme.23763 


\title{
Mary Douglas et la Bible $\mathrm{La}(\mathrm{re})$ conversion d'une anthropologue
}

\author{
Christophe Lemardelé
}

LE 16 MAI 2007, deux ans avant Lévi-Strauss, disparaissait la célèbre anthropologue britannique Mary Douglas. La presse a fait état de cette disparition (Le Monde, The Guardian), rendant hommage au travail prolifique et très original de l'ethnologue, notant au passage que sa fin de carrière fut occupée par des publications «bibliques » (livres et articles). Mais, comme dans tout hommage, le parcours est lissé au point de faire disparaittre toute aspérité, donnant à penser que la carrière fut maitrisée de bout en bout, comme suivant un fil logique ou un plan d'ensemble déterminé plus ou moins consciemment dès les premières publications. Or, la réalité est tout autre, car il y a un écart important - voire une contradiction profonde - entre Mary Douglas anthropologue et Mary Douglas bibliste.

Les spécialistes des sciences bibliques ne semblent guère s'en rendre compte - ni même le sociologue s'intéressant à ce milieu scientifique ${ }^{1}-$, tant le prestige de l'anthropologue suffit sans qu'il soit besoin de comparer pleinement ses travaux de natures différentes. De cette manière, ne pas se

1. «Seuls quelques essais d'anthropologie compréhensive tentent de rappeler le texte aux vibrations rituelles qui lui ont donné la forme d'un hymne de circumambulation, d'un récit sacrificiel ou d'un recueil utile mais fort obligeant de prescriptions détaillées », Pierre Lassave (2011: 189). L'auteur cite en note l'ouvrage de Douglas sur le Lévitique qui ne relève effectivement pas de l'anthropologie analytique mais "compréhensive" (?), tout en rappelant à juste titre que le pionnier de "l'anthropologie biblique » fut William Robertson Smith et ses Lectures on the Religion of the Semites vieilles de plus d'un siècle: d'une part, il ne fut guère suivi, d'autre part, son anthropologie, certes datée, n’était pas "compréhensive».

Cet article a pour origine une communication orale énoncée lors du congrès international IоSOT (International Organisation for the Study of the Old Testament) qui s'est tenu à Munich, en août 2013. Le titre original de la communication était : "Mary Douglas et la Bible : l'oubli de l'anthropologie». 
montrer véritablement critique vis-à-vis des derniers travaux de Mary Douglas semble être une règle non écrite chez de nombreux biblistes, règle qui permet de "certifier conformes à l'anthropologie" des travaux bibliques qui se sont faits le plus souvent en dehors du champ de réflexion comparatiste de cette discipline ${ }^{2}$. Dans un livre récent, Jonathan Klawans, spécialiste du sacrifice et des règles de pureté, pointe, quant à lui, l'«erreur» de l'anthropologue historique Francis Schmidt concernant l'usage qu'il avait fait des thèses de Douglas ${ }^{3}$. Loin d'ignorer ou de nier l'existence d'une évolution conséquente dans les interprétations de l'anthropologue britannique concernant les textes bibliques, Jonathan Klawans fait son miel des interprétations symboliques de celle-ci, mais sa thèse de l'imitatio Dei est, de notre point de vue et malgré ce qu'il affirme, bien plus à rapprocher des ultimes travaux bibliques de Douglas que de ses écrits théoriques plus anciens ${ }^{4}$.

Les anthropologues, quant à eux, semblent le plus souvent ne pas porter d'intérêt aux travaux bibliques de Douglas en les ignorant complètement (Lenclud 2000), voire, quand ce n'est pas le cas, ne pas être en mesure de les évaluer scientifiquement (voir De Heusch 2007), tant la "discipline » qu'est l'exégèse biblique ne manque pas de pièges épistémologiques. Il est vrai qu'ils ont été publiés dans des ouvrages collectifs et des revues scientifiques étrangers à leur espace bibliographique habituel et que les titres des articles et des ouvrages de Douglas consacrés à la Bible ne peuvent susciter l'intérêt anthropologique. D'ailleurs, dans ses derniers livres, l'auteur fut si peu anthropologue qu'il fallut la ruse commerciale d'un éditeur pour transformer radicalement le titre original Leviticus as Literature en L'Anthropologue et la Bible (2004a), le reléguant à la place de sous-titres.

2. Contrairement à Ronald Hendel, on ne peut assurément pas considérer que l'œuvre anthropologique de Douglas "culminating with her biblical trilogy, In the Wilderness (1993), Leviticus as Literature (1999), and Jacob's Tears (2004)» (2008:3).

3. Cf. le livre de Francis Schmidt dont le titre La Pensée du Temple (1994) fut intelligemment traduit en anglais par How the Temple Thinks (Sheffield, 2001), en référence à l'ouvrage de Mary Douglas, How the Institutions Think, puisque Schmidt tentait de s'appuyer autant sur cet ouvrage que sur Purity and Danger.

4. "Ironically, Schmidt's work depends on and develops those aspects of Purity and Danger that even Douglas herself would no longer apply to the Jewish purity and sacrificial systems. Schmidt's book appeared (in its first, French edition) in 1994, just a few years too soon to make use of Douglas's more recent and provocative works on purity. Importantly, these publications undercut her original (and more typically functionalist) contributions to the field. At the same time, Schmidt virtually ignores those aspects of Douglas's early work that have proven most helpful to us: the effort to find symbolic meanings in ritual structures. Thus in a work devoted to uncovering the thinking of the temple, we find little attention paid, if any, to notions such as imitation Dei, the divine presence, or to the cosmic symbolisms " (Klawans $2006: 109-110$ ).

5. Pour un compte rendu de cet ouvrage dans sa traduction en français, cf. Christophe Lemardelé (2007). 
Pour comprendre la trajectoire de Mary Douglas, qui la mène de l'ethnologie de l'Afrique à l'exégèse biblique, il faut revenir à sa publication phare: Purity and Danger en 1966, traduite en français dès 1971 et publiée chez François Maspero, qui fit tant pour l'anthropologie historique, sous le titre De la souillure - là encore, seul le sous-titre est traduit fidèlement : Études sur la notion de pollution et de tabou. L'ouvrage eut un écho considérable chez les anthropologues mais aussi parmi les biblistes à cause d'un chapitre devenu fameux, consacré aux prescriptions alimentaires bibliques et intitulé de manière un peu provocante "Les abominations du Lévitique».

\section{Mary Douglas socio-anthropologue}

Lorsqu'en 1966, Mary Douglas fit paraitre son essai Purity and Danger, c'était finalement prolonger celui de Lévi-Strauss, La Pensée sauvage, publié quatre ans plus tôt. En effet, dans l'un et l'autre ouvrage se manifeste une même volonté de prouver que la pensée humaine est une, sous des formes variées et riches. Ainsi, Douglas montrait que l'hygiénisme moderne, bien que fondé sur des bases scientifiques, n'était pas moins «magique» que les règles de pureté dans telle ou telle culture : l'hygiénisme moderne étant tout autant un producteur d'interdits divers pas toujours explicites et rationnels ${ }^{6}$. Elle démontrait encore que ces règles, quelles qu'elles soient et d'où qu'elles soient, sont une réponse au désordre et au malheur, et que la souillure ne peut être un phénomène isolé (Douglas 1992 [1966] : 61). Et c'est sans doute à ce niveau d'interprétation que Douglas mena ses travaux théoriques les plus ambitieux, car il s'agissait de saisir du social derrière le rituel étant donné que celui-ci fait presque toujours système. C'est pourquoi sa série de conférences publiée en 1986 avec le titre How the Institutions Think (Douglas 1999) venait apporter les fondations sociologiques à l'édifice : les institutions pensent et elles sont un savoir collectif conservateur tout en étant évolutif. La typologie et la théorie grid-group qu'elle avait énoncée dans son ouvrage de $1978^{7}$ étaient donc à même d'éclairer l'ancrage social d'une culture. Aussi, après avoir abordé et trouvé la clé de la taxonomie si particulière contenue dans deux textes bibliques, on était en droit d'attendre que l'anthropo-

6. Au passage, elle récusait, non sans une ironie mordante, toute interprétation de ce type pour expliquer un rituel ou un interdit énigmatique : "La religion comparée a toujours été infestée par le matérialisme médical» (Douglas 1992 [1966] : 49, nos italiques).

7. Cet aspect était déjà abordé dans l'ouvrage de 1970, réédité plus tard : cf. Douglas (1996a: 54-68). Concernant ces aspects de l'œuvre de Mary Douglas, cf. Marcel Calvez (2006). 
logue nous éclaire sur l'institution qui les avait produits quand elle examina à nouveau le texte du Lévitique (Douglas 1993a). Ce ne fut pas le cas et elle s'en est expliqué :

"Sachant que cette analyse était relativement nouvelle ["Les abominations du Lévitique" de 1966], j'attendais, pleine d'espoir, qu'une fois clarifié l'angle d'enquête le matériel historique biblique soit réexaminé et que soit révélé le levier social interne fourni par le concept d'impureté. Mais rien n'arriva” (Douglas 1998 : 10-11).

Il est vrai que préciser le contexte historique des textes de la Torah n'est guère facile et est toujours sujet à débat entre spécialistes, mais il faut dire aussi que Mary Douglas ne s'est pas employée à déterminer l'ancrage social de ces textes ${ }^{8}$, elle a même pris un tout autre chemin.

La brève analyse de 1966, donc "Les abominations du Lévitique " (Douglas 1992 : 61-76), qui ne visait qu’à illustrer la thèse du livre, fit tant de bruit que le livre fut bientôt connu par les biblistes pour ce seul chapitre. Quelques années plus tard, l'anthropologue fut même invitée à écrire une postface critique dans l'ouvrage d'un historien du judaïsme? Il ne fait pas de doute que l'apport de Mary Douglas aux «sciences bibliques " a été considérable. Cet apport a d'ailleurs été bien plus important que ne le fut celui de René Girard ${ }^{10}$, car l'analyse prenait, si l'on peut dire, les biblistes par surprise et à revers. En effet, Douglas interprétait les deux textes du Lévitique et du Deutéronome sans complaisance et proposait une explication lumineuse et non une théorie générale teintée d'accents apologétiques. Pour la première fois, il ne s'agissait pas de comprendre les données bibliques à l'aune d'une anthropologie générale de cabinet - à la Frazer - mais bien du passage de ces données au tamis de l'anthropologie sociale fondée sur l'ethnographie de terrain. Finalement, ce que fit Douglas en intégrant les interdits bibliques à sa démonstration est quelque peu parallèle à ce que certains hellénistes firent dans la mouvance de Jean-Pierre Vernant s'inspirant du structuralisme: les anciens "Israélites " pas plus que les Grecs de l'Antiquité ne sont à nous et ne sont nous (Detienne 2005), et s'ils ne peuvent être à même de nous renseigner sur notre culture ${ }^{11}$, ils peuvent au contraire enrichir le savoir

8. Ni même l'ancrage culturel plus large, à la différence tout de même de Walter Houston (1993).

9. Ce dont elle ne se priva pas de faire, notamment en parlant d'approche historique avec des inclinations positivistes, cf. Douglas (1973).

10. «Les travaux de Mary Douglas sur le pur et l'impur, ceux de René Girard sur le sacrifice ou encore le bouc émissaire ont attiré l'attention sur le Lévitique où ces thèmes occupent une place prépondérante", Alfred Marx (2011:9).

11. Douglas abandonnera cette distance prudente par la suite: «My personal project in the study of the Bible is to bring anthropology to bear on the sources of our own civilization " (Mary Douglas citée in Hendel 2008: 3, nos italiques). Ronald Hendel reprend ici un article de Mary Douglas "Why I Have to Learn Hebrew : The Doctrine of Sanctification» (2004c). 
anthropologique de l'humanité par leurs spécificités culturelles relevant encore de "sociétés froides". L'anthropologue britannique a donc ni plus ni moins mis sur le même plan les tabous bibliques et ceux des Lele d'Afrique centrale, ce qui ne pouvait que choquer les exégètes des textes sacrés. Mais comme elle trouva la clé de l'énigme, l'interprétation fit date et fut progressivement acceptée.

La liste exhaustive des animaux interdits contenue en Lévitique 11 - qui reprend, développe et affine celle de Deutéronome 14 - n'intéressait guère les exégètes avant que Mary Douglas n'en révèle la cohérence. En effet, elle rebutait l'esprit empreint de rationalité théologique quelque peu hermétique à la notion d'impureté physique. Et puis, tout semblait arbitraire dans cette liste. En lisant attentivement le texte, le prenant au sérieux et ne le rejetant pas comme étant le produit d'une pensée magique, familière qu'elle était des tabous culturels de par son ethnographie africaine, l'anthropologue établit clairement deux critères déterminants classant les animaux en espèces pures et impures. Le premier est énoncé dans le texte même, le second en est déduit à partir d'une réflexion sur la sainteté, ou la sacralité, si présente dans ces textes :

«Être saint, c'est être entier, être un ; la sainteté, c'est l'unité, l'intégrité, la perfection de l'individu et de ses semblables. [...] [S] euls sont purs les animaux qui sont entièrement conformes à leur classe" (Douglas 1992 [1966] : 73-74).

Ainsi était établi le caractère imparfait des animaux prohibés : le premier critère concernait les animaux terrestres herbivores qui ne peuvent être parfaits que s'ils ruminent et ont un sabot fendu; le second critère permettait d'écarter les animaux non adaptés à leur élément naturel : "Dans le firmament, ce sont les volatiles ailés à deux pattes; dans les eaux, les poissons à écailles qui nagent avec des nageoires; sur la terre, ce sont les animaux à quatre pattes et qui sautent ou marchent. Tout groupe de créatures non équipées pour le mode de locomotion qui lui est imparti dans son élément, est contraire à la sainteté » (Ibid. : 74), ce qui exclut de fait les espèces terrestres rampantes. De cette manière, la taxonomie présente dans ce texte donnerait une idée précise des animaux parfaitement conformes à leur milieu de vie, n'ayant aucun caractère hybride ou évolutif - la nageoire développée et décollée du corps du fameux cœlacanthe l'aurait peut-être classé comme suspect parmi les poissons, mais qu'auraient pensé alors les auteurs de ce texte d'une espèce comme l'ornithorynque? Évidemment, ainsi vue, cette taxonomie traduit une vision fixiste des espèces, mais elle suggère aussi que la création du monde et des êtres vivants, telle qu'elle est narrée au tout début du livre de la Genèse, était imparfaite. Cela n'est d'ailleurs pas en contradiction 
complète avec le texte ouvrant la Bible, car il y a bien longtemps que l'on a souligné le fait que le verbe utilisé pour désigner l'action divine n'est pas à traduire par «créer ", mais par « séparer ».

En mettant en évidence la perfection d'espèces vivantes et le caractère imparfait d'autres, Mary Douglas ouvrait donc le texte à la compréhension de tous, sans toutefois s'attacher alors à l'ancrage historique et social d'une telle classification. Elle reconnaissait seulement que, vraisemblablement, suivant un éminent bibliste quelque peu ancien (Samuel Rolles Driver), « les prescriptions alimentaires entérinent a posteriori un état de fait » (Ibid.), mais sans aller au-delà de l'hypothèse que ces prescriptions avaient pour origine une vie pastorale excluant de fait des animaux comme le porc ${ }^{12}$. L'anthropologue Mary Douglas privilégiait l'étude synchronique pour mettre à jour un système symbolique. Lorsque Jacob Neusner lui opposa sa lecture diachronique insistant sur une propagande sacerdotale spécifique, propre à la Torah et ne pouvant donc pas être étendue au judaïsme rabbinique ultérieur, Douglas répondait vertement que pour comprendre des règles de pureté, il était nécessaire de tenter de comprendre un monde constitué (Douglas 1973 : 139), donc un système symbolique cohérent et subtil. En effet, si on ne peut considérer autrement Lévitique 11 que comme étant un texte idéologique, il n'émane pas pour autant d'un groupe religieux exogène ou révolutionnaire voulant à toute force imposer son système. Le fait que la classification soit connue dans un texte plus ancien et moins développé (Deutéronome 14) prouve assez que le système symbolique existait avant la mise par écrit des règles en place. Cette mise par écrit pour faire d'un texte force de loi n'a pu que pousser la logique du système à son maximum, sans que ce fût pour autant une nouveauté complète. Que Mary Douglas revînt plus de deux décennies plus tard sur ces prescriptions bibliques aurait pu faire penser qu'elle voulait finir le travail commencé en étudiant le système symbolique en relation avec son ancrage social. Même si son objectif put être celui-là, ses nombreux travaux bibliques prirent toutefois une orientation toute différente.

12. Parmi les critiques qui affleurèrent contre son interprétation, il y en eut qui exprimaient de manière plus ou moins nette leur scepticisme face à la moindre importance accordée au tabou du porc, obligeant Douglas à s'en expliquer : "The pig is not singled out for special abhorrence more than the camel and the rock badger. The diatery rules, I suggested, should be taken as a whole and related to the totality of symbolic structures organizing the universe " (1996a [1970] : 38). Même si, peu après, en reprenant l'étude du Lévitique, elle assouplit sa position en reconnaissant une spécificité potentielle du porc, elle ne renonçait pour autant pas à l'interprétation taxonomique (1975 : 272). D'ailleurs, la neutralité du texte biblique est corroborée par l'historiographe judéen Flavius Josèphe qui mentionne une proclamation officielle du roi Antiochos III devant s'appliquer à Jérusalem à partir de 200 av. J.-C. Or les règles de pureté y sont rappelées ainsi qu'une brève et hétéroclite liste d'animaux interdits (cheval, mulet, âne, léopard, renard, lièvre) sans que le porc soit mentionné (Antiquités juives 12, 145-146). 


\section{Philologie versus anthropologie}

Quand Mary Douglas publia de nouveau un article sur les animaux prohibés - cette fois dans une revue biblique! -, un philologue spécialiste de l'hébreu ancien venait de publier le premier de ses trois volumes de commentaire du Lévitique et il y relevait les nombreuses erreurs philologiques de l'anthropologue (Milgrom 1991: 720-721) ${ }^{13}$. C'est sans doute la raison pour laquelle celle-ci décida d'apprendre l'hébreu, de manière à ne plus dépendre d'une traduction moderne et pour accéder à la rigueur philologique de l'exégète biblique. Son article de 1993 commence d'ailleurs par une note remerciant Jacob Milgrom pour son aide, ce qui reflète un état d'esprit de l'auteur tout à fait différent de celui qu'elle avait adopté vingt ans plus tôt vis-à-vis de Jacob Neusner : le regard éloigné de l'anthropologue révélait sa faiblesse, après avoir été un atout, lorsqu'il s'était agi d'analyser précisément le vocabulaire hébraïque. Le même Milgrom écrivit dans la foulée de son premier volume de commentaire un article précisant et développant l'analyse de la complexité philologique de Lévitique 11 , de manière à pouvoir proposer une nouvelle interprétation d'ensemble (1992). Cet article et les remarques critiques du spécialiste du Lévitique auront un effet considérable sur les publications ultérieures de Douglas: d'une part, elle s'efforça de maitriser le vocabulaire en langue originale, varié et toujours difficile à traduire, ensuite, elle reconnut ses erreurs passées au point de revenir radicalement sur sa première interprétation (Douglas 1996b; 2000). On peut même se demander si l'influence de Milgrom ne se transforma d'ailleurs pas en une réelle dépendance de Douglas vis-à-vis de ses travaux puisque l'anthropologue adopta l'ensemble des points de vue du philologue sur les textes sacerdotaux de la Bible. D'une certaine manière, en apprenant l'hébreu, Mary Douglas était redevenue une étudiante suivant les thèses d'un maître qualifié sur la question, au savoir linguistique incontestable.

Pour Jacob Milgrom, il y avait des distinctions d'importance à opérer, notamment entre les espèces réellement impures et celles qualifiées d'abominables. Mais tout d'abord, d'un point de vue strictement philologique, et à la différence de Mary Douglas qui traitait indistinctement des animaux abominables et des animaux impurs, Milgrom faisait remarquer qu'il y avait d'un côté les animaux dits țâmé ( «impurs») et de l'autre des espèces dites šeqes. Il précisait en outre que ce dernier terme a la signification de "détestable", ce qui a pour effet de disqualifier la traduction

13. Les erreurs ne se trouvent d'ailleurs pas tant dans "Les abominations du Lévitique " que dans «Deciphering a Meal» (Douglas 1975 : 249-275), article publié auparavant dans la revue Dedalus en 1972. 
du terme par "abomination", qui recouvre en revanche le terme hébraïque tốébāh, absent de Lévitique 11 mais présent en tant que synonyme d'impur dans le texte parallèle sur ces règles alimentaires qu'est Deutéronome 14. Ainsi, il n'y a pas d'abominations dans le Lévitique, il y a en revanche une dichotomie entre des animaux impurs et des animaux détestables à rejeter de l'alimentation. Cet éclairage philologique est bien entendu nécessaire, il permet de faire la distinction entre des animaux qui appartiendraient à un premier cercle et d'autres issus d'un cercle plus éloigné. Mais la rigueur philologique n'aboutit pas pour autant à l'invalidation de l'interprétation de Douglas. En effet, non seulement ces espèces détestables ne peuvent faire partie de l'alimentation, mais leur cadavre ne peut pas plus être touché que celui des espèces impures (Lévitique 11,8 et 23-24) ${ }^{14}$. Dans ce cas, les erreurs philologiques de Mary Douglas n'ont guère de conséquence puisque les deux "classes " d'animaux, sans être totalement similaires, partagent les mêmes interdits. Si l'on doit faire une distinction de cet ordre, c'est entre les animaux proprement dits et ce que le texte appelle «bestiole " (̌̀̀rès), ce qui semble correspondre à un second cercle effectif d'espèces vivantes, petits mammiferes, sauriens et insectes, qu'ils soient aquatiques, ailés ou pullulant sur la terre. Surtout, la discussion et la mise au point philologiques ne remettent aucunement en cause le schéma interprétatif de l'anthropologue étant donné que le caractère imparfait de certaines espèces est toujours ce qui est le mieux à même d'expliquer leur impureté ${ }^{15}$. Malgré cela, Mary Douglas renoncera à sa clé d'interprétation. Dans son article de 1993, elle en fait à peine mention, sinon pour souligner le paradoxe biblique faisant concilier la sacralité du sang de tout animal et l'impureté physique de certains (1993a: 4-5). Dans sa communication de 1996, elle reconnaît ses erreurs de lecture et d'interprétation en relevant la richesse du vocabulaire hébraïque pour désigner ce qui est de l'ordre de l'impureté et de la transgression (1996b : 88). Et, au lieu de tenter de comprendre un système de pensée anthropologique se développant du

14. Même si le texte du Lévitique se veut rationalisant, il ne parvient toutefois pas à être totalement cohérent. C'est pourquoi Milgrom le force quelque peu en écrivant que «Whatever is țāme transmits impurity by touch ; whatever is šè ès may not be eaten" (1992: 108).

15. L'imperfection des espèces impures semble indiquer un mélange ou une confusion de caractères physiques, ce qui se retrouve ailleurs dans le Lévitique : "Gardez mes lois : n'accouple pas deux espèces différentes de ton bétail ; ne sème pas dans ton champ deux semences différentes; ne porte pas de vêtement en étoffe hybride, tissée de deux fibres différentes » $(19,19)$, précepte qui recoupe et développe celui de Deutéronome 22, $11:$ :Tu ne t'habilleras pas avec une étoffe hybride de laine et de lin " (traduction Тов [Traduction œcuménique de la Bible]). Ce type de tabou se retrouve dans le judaïsme orthodoxe contemporain qui prohibe tout mélange de produits lactés et de produits carnés au point d'engager des couples modernes à respecter strictement ce principe de séparation, cf. Laurence Faure (2010). 
texte plus simple du Deutéronome au texte du Lévitique, Douglas se concentra sur ce dernier livre afin de mettre en évidence une pensée théologique spécifique, fondant notre culture et échappant au comparatisme.

L'analyse philologique ne mène pas forcément à de grandes découvertes mais Jacob Milgrom, dans son article, en retirait d'importantes déductions : les animaux "détestables» étaient, pour lui, seulement interdits pour l'alimentation, mais, en plus, puisqu'ils n'étaient pas dits "impurs", c'est qu'ils étaient purs (1992: 109)... Or le texte ne permet pas une telle interprétation. La dichotomie sacerdotale séparant le monde entre le pur et l'impur ne peut prétendre caractériser tout le réel, d'où l'existence de failles dans la pensée sacerdotale. Toutefois, poursuivant dans cette logique systématique, l'exégète en venait à comprendre Lévitique 11 à la lumière du récit de la Création (Genèse 1), puisque les deux textes évoquent les trois éléments primordiaux que sont la terre, le ciel et l'eau, et, dans la nécessité qu’il était de prouver que les animaux dits détestables étaient purs, il affirmait qu'ils l'étaient parce qu'ils émanaient de l'élément pur par excellence : l'eau (Ibid: 110-111). Or, si les oiseaux et les bestioles ailées ne vivent pas dans cet élément, ils n'en proviennent pas plus ${ }^{16}$. Certes, les parallèles entre les deux textes sont frappants, pour autant, on ne peut totalement les calquer l'un sur l'autre. En effet, Genèse 1 ne parle pas de " bestioles" pour les petites bêtes vivant sur la terre et Lévitique 11 utilise le terme šerès indifféremment pour des bestioles aquatiques et terrestres ${ }^{17}$. Par ailleurs, Milgrom donnait la priorité au texte de la Création mais rien ne dit qu'il fut élaboré en premier. Au contraire, les auteurs de ce texte ont pu s'appuyer sur la taxonomie si développée de Lévitique 11 en fonction des trois éléments. Les exégètes bibliques savent en effet d'expérience que la rédaction des textes et des livres bibliques le fut au détriment de la linéarité des récits. Le cas le plus connu est justement ce récit de Création considéré comme ultérieur au second récit narrant la faute d'Adam et Ėve (Genèse 2-3 $)^{18}$. De même, Milgrom remarquait que le texte parallèle du Deutéronome ne faisait pas de distinction entre animaux impurs et bestioles détestables, supposant que le terme tâmé' avait totalement

16. Jacob Milgrom s'appuie sur Genèse 1, 20 pour affirmer cette provenance, mais le passage ne fait qu'indiquer l'ordre de création des animaux : d'abord les poissons et les oiseaux, ensuite les espèces terrestres.

17. Dans l'ensemble Genèse 1-9, de la Création à la re-Création après le Déluge, il est question de petites bêtes remuantes (rèmès), qui ne sont ni l'objet de restrictions alimentaires ni stigmatisées pour leur aspect. Il y a donc pluralité des textes et des contextes - au «système » inclusif du mythe cosmogonique s'oppose la classification rituelle excluante.

18. Traditionnellement, on considère qu'il y eut un récit pré-sacerdotal élaboré en deux temps, récit auquel on aurait ajouté le poème cosmogonique de Genèse 1: cf. Christoph Uehlinger (2004: 120-123). 
"supplanté» le terme šeqes, alors que l'on peut bien plus facilement défendre l'inverse : le texte de Lévitique 11 a vraisemblablement pour modèle Deutéronome 14, plus ancien, pour préciser la taxonomie, c'est pourquoi il est plus long et plus riche lexicalement. Il en résulte que la philologie si rigoureuse de l'exégète était fondée sur une vision conservatrice de l'élaboration des textes bibliques et que la bonne démarche historique aurait été d'analyser «les abominations du Deutéronome » avant celles du Lévitique, tout en étudiant le vocabulaire comme étant la marque de l'évolution d'une pensée, non comme procédant de catégories intangibles qui auraient été mises en place dès le récit de Création. La question est de savoir comment une anthropologue comme Mary Douglas, partie de son interprétation de 1966, put arriver à une telle convergence de vues avec Milgrom qu'elle en renia ses premiers travaux. Car considérer les espèces détestables comme pures change le paradigme, ce ne sont plus des espèces à rejeter, indignes du dieu créateur, mais à respecter, voire à protéger en interdisant aux hommes de les manger. Or, c'est bien à la conclusion à laquelle est arrivée Douglas (2004a : 196).

\section{Mary Douglas bibliste}

La «seconde» Mary Douglas differe de la première en ceci : au lieu d'évoquer, pour la comparaison, des données ethnographiques, elle s'appuyait sur des textes bibliques parfois très éloignés de ceux qu'elle analysait. C'était déjà le cas dans son article de 1993 puisqu'elle commentait d'abord un passage du livre d'Isaïe pour parler ensuite du Lévitique et de ses animaux interdits. C'est là une méthode d'exégète biblique. Or Isaïe 1, 10-17 n'est rien de plus qu'un passage dans le style des prophètes pour dénoncer les offrandes sacrificielles effectuées avec un esprit d'impiété. S’il est vrai que ce texte réprouve la violence des hommes et leurs mains pleines de sang, il est bien imprudent d'établir une analogie entre des victimes humaines et animales en concluant que la sainteté se révèle incompatible avec le caractère prédateur de certaines espèces (Douglas 1993a : 22-23) ${ }^{19}$. Mary Douglas ne s'intéressa guère à la théorie du sacrifice, d'un point de vue biblique comme d'un point de vue anthropologique, mais ses conceptions sur les animaux prohibés ont suivi en parallèle l'herméneutique théorique de Jacob Milgrom et d'Alfred Marx : le sacrifice animal biblique serait par essence non violent et l'alimentation humaine végétarienne à l'origine (Lemardelé 2011-2012). Comme eux,

19. Il est d'ailleurs à noter qu'à l'exception d'une liste d'oiseaux prédateurs, les espèces carnivores terrestres ne sont pas mentionnées. 
elle prenait appui sur Genèse 9 et Lévitique 17 dans lesquels l'idéal non violent serait concentré dans l'interdit d'ingérer du sang ${ }^{20}$. Au lieu de comprendre un tabou anthropologique dépassant largement la Bible ${ }^{21}$, Douglas en faisait, comme eux encore, une spécificité éthique en prenant au pied de la lettre le début de Genèse 9, texte dans lequel le dieu suprême déclare établir son alliance avec les animaux pour ne plus chercher à les exterminer par le Déluge - texte donc à saisir uniquement dans son contexte littéraire. Ainsi, dans ses diverses publications liées aux textes bibliques, une question ingénue sous forme de problématique est, comme un leitmotiv, sans cesse posée : comment le Créateur qui a créé toutes les espèces peut-il en exclure certaines en les qualifiant d'impures (Douglas 1998: 5-6)? Reprenant quelque peu le mode de raisonnement de Milgrom, elle s'interrogeait sur la raison de la neutralité de Lévitique 11 qui n'énonce pas de cause à l'impureté de ces espèces. Mais cette neutralité n'est pas réelle car dire d'espèces animales qu'elles sont détestables suffit bien à les caractériser négativement et à les considérer en dehors de la Création qui n'est pas tant, rappelons-le, une création ex nihilo qu'une mise en ordre du chaos originel.

Mary Douglas ne s'est pas contentée de revenir sur la question des animaux interdits, elle a également développé sa réflexion sur la souillure. Mais au lieu d'en faire un révélateur sociologique, elle en fit une émanation du sacré dans la Bible ${ }^{22}$. Analysant les exemples de lèpre, elle en concluait qu'il s'agissait d'une contagion sacrée, ce qui n'est guère contestable étant donné que des exemples narratifs indiquent une nette correspondance entre une souillure manifeste et un fléau divin. Le problème est qu'elle réduisait le "monde biblique " à deux sphères : le monde profane des humains et le monde du sacré, source illimitée du pouvoir pour le bien comme pour le mal (Douglas 1996b: 91). Prenant le monothéisme biblique comme un élément assuré du réel, elle ne pouvait considérer que, malgré l'affirmation d'un seul culte pour une seule divinité, l'impureté continuait de provenir de l'Underworld. La preuve en est fournie par l'existence d'une entité divine dissimulée dans le grand rituel du Yom Kippur (Lévitique 16). Mary Douglas a d'ailleurs publié deux études sur le sujet

20. Dans ces deux textes est répété, pour légitimer un tabou très ancien, que la vie est dans le sang. Après avoir répliqué à Jacob Neusner qu'une collection de métaphores ne constituait pas nécessairement un système symbolique (Douglas 1973 : 138), Mary Douglas prit cette métaphore au pied de la lettre.

21. Commun avec l'islam, mais vraisemblablement pré-islamique, il convient de souligner que «le sang est source de tous les dangers, attirant les jnûn et les esprits néfastes ", Pierre Bonte (1999: 40). 22. Sacré dont elle donnait encore une bonne définition sociologique dans l'introduction de 1996 de Natural Symbols: "the idea of the dangerous and powerful Sacred is indeed formed by living together and trying to coerce one another to conform to a moral idea " (Douglas 1996a [1970] : XVI). 
(2002, 2003), mais en voulant à toute force distinguer le rite du bouc émissaire de rites apotropaïques grecs. L'argumentation n'est guère convaincante car il s'agit de lire sans esprit critique un texte biblique qui veut que le bouc emmené dans le désert, mené dans un «lieu» nommé Azazel, fût rendu à sa liberté 23 . Or le texte rabbinique du traité Yoma de la Mishna ne laisse aucun doute sur la destination du bouc: malmené à sa sortie par des dévots excités, qui lui arrachaient des poils et le maudissaient, l'animal était mené par un homme pour être précipité violemment dans un ravin du désert. En outre, Douglas devait procéder à une étymologie fantaisiste d'Azazel pour donner une interprétation du nom cohérente avec sa thèse ${ }^{24}$. Il est vrai qu'il y a un problème avec ce terme car les auteurs du texte ne se sont pas contentés de dissimuler la finalité du rite, ils ont également modifié la graphie du destinataire. Comme Azazel intervient dans des textes un peu plus tardifs, dont le traité Yoma de la Mishna, on peut facilement rétablir la graphie d'origine: "azaz " et "'el », c'est-à-dire le dieu fort. Ainsi, le bouc portant toutes les fautes de l'année n'était pas gentiment accompagné dans le désert mais voué à une divinité des Enfers. Les exemples d'oiseaux relâchés librement - en fait, sans destinataire après un rituel de purification se comprennent de la même manière : les auteurs des textes ne devaient fabriquer du rituel qu'en fonction d'une divinité unique qui ne pouvait à elle seule détenir toutes les fonctions religieuses. C'est d'ailleurs cette non-prise en compte de la monolâtrie stricte contenue dans les textes, qui a conduit Douglas à penser que la doctrine du monothéisme condamnait toute forme de magie et de vaine superstition (2004b: 194) ${ }^{25}$, sans prendre en considération la question d'un «monopole» cultuel des prêtres yahwistes (Schmitt 2008 : 9). Malgré une volonté affichée de rechercher un système de pensée dans le Lévitique et plus largement dans la Bible, on est bien obligé de conclure que la "seconde" Mary Douglas ne fit rien d'autre que conforter l'idéologie de ces textes en ne les passant plus au crible de l'analyse anthropologique ${ }^{26}$ - la notion même de culture qui lui était chère ${ }^{27}$ disparaissait.

23. Que penser de ce type de phraséologie : «le Dieu du Lévitique n’est pas punitif mais miséricordieux " (Douglas 2002: 130) ?

24. Litt. : ez, le "bouc», et azal, «s'en aller» (Ibid.: 131).

25. Rüdiger Schmitt commente ainsi la citation entière de Douglas : "This position fits into the perception of magic, divination and other forms of communication with the dead in mainstream Old Testament scholarship. Remarkably, this is exactly the way of arguing that Douglas criticized so much in her earlier writings, in particular in Purity and Danger" (2008 : 4).

26. Comme l'écrit encore Rüdiger Schmitt : «she [...] simply equates the theology promoted in the texts with socio-religious reality" (Ibid.: 12).

27. Au point de confronter sa théorie culturelle grid-group aux problématiques économiques et de pouvoir modernes stigmatisant culturellement des nations pauvres : Douglas (2007). Ainsi, .../... 


\section{Théologie versus anthropologie}

Nous l'avons déjà entrevu, le «retour» de Mary Douglas dans le domaine des études bibliques - nous devrions dire sa venue aux sciences bibliques - dans les années 1990 s'est visiblement fait sous les bons auspices de Jacob Milgrom. Ce dernier avait publié un nombre considérable d'articles faisant date sur le système rituel biblique avant la parution de son commentaire en trois volumes du Lévitique. S'il a grandement défendu une approche philologique rigoureuse dans un cadre que l'on peut considérer comme étant d'anthropologie historique, en revanche, ses interprétations au terme de l'analyse convergeaient en général vers la recherche d'une éthique propre à la Torah. Comme il le raconte lui-même dans la préface d'un ultime et condensé commentaire du Lévitique (Milgrom 2004: XI-XIII), tout exégète avait jusqu'alors tendance à s'écarter des lois rituelles contenues dans ces textes. De manière assez intuitive, il s'engagea dans la voie de l'étude de ces rituels dès 1963 avec un premier article intitulé "The Biblical Diet Laws as an Ethical System » (Milgrom 1983 : 104-118). Il fut par la suite, toujours selon lui, influencé par Purity and Danger et par d'autres travaux anthropologiques mais sans jamais renoncer à sa quête d'une éthique : "I have discovered that the rituals in Leviticus contain fundamental values that in aggregate prescribe a holy and ethical life»(Milgrom 2004: XIII). Il semblerait que sa parfaite connaissance de l'hébreu et des textes bibliques ait directement influencé Mary Douglas tant elle a adhéré à ses thèses éthiques principales concernant la sacralité du sang de tout être qui conduisait à ne tuer par le sacrifice que les bêtes pures et à protéger les autres ${ }^{28}$. C'est en tout cas ce que l'on devine en lisant la lettre d'hommage écrite par Milgrom en 2001 pour les quatre-vingts ans de Douglas et publiée par Ronald Hendel. Après avoir dit que l'anthropologue avait eu une grande influence sur son propre travail, il remarque comme il l'a plus souvent et plus favorablement citée tout au long de ses trois volumes de commentaire du Lévitique. Or, comment explique-t-il cette évolution? Par le fait que Douglas «studied Biblical Hebrew assiduously and honed her knowledge of pentateuchal criticism»(Hendel 2008: 14). Autrement dit, quand les travaux de

[Suite de la note 27], il n’y eut pas tant une évolution de pensée définitive chez Mary Douglas que la cohabitation de deux pensées, l'une anthropologique, développant toujours plus une théorie de la culture, l'autre biblique et relativement hermétique à la première - il n'y eut pas véritablement de "contagion» entre elles. Finalement, fidèle à sa conception de la culture d'un individu comme étant quelque chose de socio-psychique, communautaire et individuel, la réflexion anthropologique relevait de la participation à la communauté scientifique tandis que la réflexion biblique était le propre de l'individu Mary Douglas.

28. "Humans have a right to nourishment, not to the life of others. Hence the blood, which is the symbol of life, must be drained and returned to the universe, to God», Jacob Milgrom (2004: 103). 
l'anthropologue ne furent plus, précisément, des travaux anthropologiques, quand ceux-ci s'accordèrent avec les siens et quand il put apparaître comme le maître indépassable ès sciences bibliques. Certes, on peut penser que l'inflexion intellectuelle de Mary Douglas vis-à-vis de la Bible ait pu provenir de son catholicisme traditionnel comme le rappelle Luc de Heusch (2007: 215), mais l'on ne peut écarter l'hypothèse d'une autorité intellectuelle déployée par un Jacob Milgrom s'érigeant en détenteur de $l^{\prime}$ hebraica veritas ${ }^{29}$. La conclusion de tout cela est que "Les abominations du Lévitique» furent une formidable intrusion de l'anthropologie dans le champ biblique qui fit considérablement avancer la recherche. Pour autant, la théologie a fini par reprendre ses droits de par la volonté du rabbin Milgrom - au sens propre du terme - d'attribuer de singulières et hautes valeurs éthiques à ces textes ${ }^{30}$. En effet, après avoir entrevu une "pensée sauvage» classificatoire dans Lévitique 11, nous sommes revenus à un certain conformisme théologique par le biais d'une éthique que n'avait pas particulièrement remarquée le maître en la matière et bon connaisseur du texte biblique en langue originale qu'était Spinoza la Bible y gagne sans doute, pas la connaissance anthropologique du Proche-Orient ancien ${ }^{31}$.

\section{Excursus : la taxonomie biblique}

Si nous reprenons rapidement l'analyse de la taxonomie du Deutéronome et du Lévitique, nous pouvons facilement déterminer que le premier texte considère qu'il y a quatre classes d'animaux, tandis que le second semble en déterminer cinq ${ }^{32}$ :

29. En 1993, Douglas écrivait ainsi : "The towering figure in the interpretation of the priestly work is Jacob Milgrom, who has been my friend for more than twenty years [...]. My overwhelming personal indebtedness shows in the large file of careful notes that I have received from him, some from work, some written while packing for a holiday, some, alas, from a hospital bed. Academics do not normally take such pains for ignorant enquirers, and I sense that I have been the beneficiary of a kind of pastoral concern " (1993b : 16, nos italiques).

30. Dans une récente monographie, un bibliste de renom, après avoir conclu que l'interprétation de 1966 était tout simplement fausse, n'en reconnaissait pas moins que les travaux ultérieurs qui s'avèrent pertinents sur la question des animaux impurs partent tous de cette interprétation. Par ailleurs, il récusait toute validité à l'hypothèse éthique de Milgrom et de Douglas, concernant la nécessité de préserver par l'interdit tous les animaux de la Création, mais tout en lisant Lévitique 11 en fonction des origines non violentes et végétariennes de la Genèse, accordant donc quelque crédit à l'approche éthique du premier..., cf. Christophe Nihan (2007 : 324-339).

31. "Douglas's claim that Israel's symbol system was fundamentally different from those of its ancient Near Eastern environment seems to be apologetic " (Schmitt 2008 : 12). Cette décontextualisation anthropologique, pour ne prendre en compte que le texte canonique comme système religieux, se retrouve dans l'ouvrage de Mohamed Benkheira (2000), qui rejette vivement le premier type d'interprétation de Douglas mais s'accorderait sans doute avec le second.

32. Penser qu'il y en a huit dans le Lévitique revient à prendre trop en compte les répétitions du texte: cf. Milgrom (1992: 107). 


\begin{tabular}{|c|c|c|}
\cline { 2 - 3 } \multicolumn{1}{c|}{} & Deutéronome I4 & Lévitique I I \\
\hline $\begin{array}{c}\text { Classe I } \\
\text { Lesimaux terrestres }\end{array}$ & $\begin{array}{c}\text { ils ruminent et ont le sabot } \\
\text { fendu en deux }\end{array}$ & $\begin{array}{c}\text { ils ruminent et ont le sabot fendu; } \\
\text { les quadrupèdes qui marchent sur la } \\
\text { plante des pieds sont impurs (v. 27) }\end{array}$ \\
\hline $\begin{array}{c}\text { Les animaux aquatiques } \\
\text { Classe 2 }\end{array}$ & $\begin{array}{c}\text { ils ont des nageoires } \\
\text { et des écailles }\end{array}$ & $\begin{array}{c}\text { ils ont des nageoires } \\
\text { et des écailles }\end{array}$ \\
\hline $\begin{array}{c}\text { Classe 3 } \\
\text { Les oiseaux }\end{array}$ & $\begin{array}{c}\text { liste d'animaux impurs } \\
\text { sans critère }\end{array}$ & $\begin{array}{c}\text { liste presque similaire } \\
\text { sans critère }\end{array}$ \\
\hline $\begin{array}{c}\text { Classe 4 } \\
\text { Les bestioles ailées }\end{array}$ & elles sont toutes impures & $\begin{array}{c}\text { alles sont impures, } \\
\text { ànception des sauterelles, } \\
\text { grillons et criquets }\end{array}$ \\
\hline $\begin{array}{c}\text { Les bestioles qui pullulent } \\
\text { sur la terre }\end{array}$ & & \\
\hline
\end{tabular}

Sur ce tableau, nous voyons bien comme les deux textes sont interdépendants et comme la taxonomie du Lévitique développe celle du Deutéronome. Apparemment, les petites bêtes au départ toutes impures pouvaient être propres à l'alimentation. On voit donc que le système symbolique recouvre des habitudes alimentaires - en premier lieu les herbivores ruminants comme les ovins et les caprins ${ }^{33}$ - qu'il s'agit d'intégrer. Par exemple, on pourrait déterminer que les oiseaux impurs le sont parce qu'ils sont carnivores, voire sont des charognards, mais les carnivores terrestres ne sont pas évoqués et l'interdiction de manger de la cigogne et du héron peut tout aussi bien relever d'habitudes alimentaires ancestrales. Il en est probablement de même pour les sauterelles et les criquets pour lesquels le Lévitique fait une exception. On voit par ailleurs qu'il y a bien un classement selon que les espèces sont conformes ou non à leur classe. C'est particulièrement le cas pour les herbivores et les

33. Exclu de la liste des animaux purs, donc comestibles, le chameau n'était visiblement pas suffisamment familier pour être intégré dans le « système biblique » alors qu'il fut admis à la «table islamique " prônant des principes similaires de pureté : prohibition du sang, du porc et des bêtes crevées (Coran, sourate II, 168 et V, 4). 
poissons ${ }^{34}$. Mais l'exclusion totale des bestioles (š̀rès ) dans le Deutéronome, puis partielle dans le Lévitique, signifie également quelque chose. Et c'est le Lévitique moins catégorique qui nous fournit un élément de réponse: il ne faut pas manger les bêtes qui marchent sur la plante des pieds et les bestioles qui pullulent sur la terre ferme. Parmi ces dernières, il y a la taupe et toutes sortes de lézards, donc des animaux de petite taille qui vivent à même le sol, voire vivent dans le sous-sol. Ce sont, pour le moins, des espèces qui ont un aspect chtonien. Or, le système cultuel du Lévitique reposait sur une conception encore apotropaïque du sang (Levine 1974: 67-91) - c'est bien pourquoi il ne fallait surtout pas l'ingérer -, le sang du sacrifice de purification, et de ce seul sacrifice, étant jeté à la base de l'autel (Lemardelé 2002). On peut donc en conclure que les deux textes taxonomiques opèrent également une distinction de base entre de grands animaux, qu'ils soient conformes ou non à leur classe, et des petites bêtes pour l'essentiel impures à cause de leur appartenance au sol et au sous-sol. Ainsi, le caractère imparfait "animal terrestre sans pattes ", opposé à la perfection du divin et donnant lieu à classification, recouvre une conception plus ancienne de l'impureté.

Le système symbolique décrit par Mary Douglas s'est donc développé sur la base d'habitudes alimentaires ancestrales, plus ou moins facilement intégrables dedans, et en lien avec des conceptions traditionnelles de l'impureté, revalorisées d'ailleurs par le Lévitique qui insiste tant - à la différence du Deutéronome uniquement centré sur l'alimentation! sur l'interdit de toucher le cadavre des espèces prohibées ${ }^{35}$, impureté contagieuse continuant de provenir d'un Underworld sans entité à cause du culte institué pour une unique divinité. La comparaison entre le Yom Kippur biblique et le rite annuel de purification du temple de Babylone

34. Même si le système recoupe en partie le mode alimentaire, il nous paraît difficile toutefois de le réduire à cela : cf. Christophe Nihan (2007 : 330-331). Le célèbre interdit de cuire le chevreau dans le lait de sa mère, qui clôt d'ailleurs la taxonomie de Dt 14, relève sans doute de ce système symbolique prohibant toute confusion, tout mélange pouvant s'avérer dangereux - ce qu'une culture peut exprimer assez paradoxalement comme ce qui est " contre-nature »-, et érigeant donc des principes structurels de séparation.

35. C'est pourquoi, à la différence de Walter Houston (1993 : 218-258), nous voyons plus l'affirmation d'une idéologie spécifique dans ces textes plutôt que le développement d'une théologie, idéologie basée sur un culte exclusif - une monolâtrie et non un réel monothéisme - et un impératif de pureté qu'on ne peut sans doute pas dissocier de préoccupations identitaires. Car, finalement, le texte de Lévitique 11 est la mise en pratique du leitmotiv de ce que les biblistes appellent la "Loi de sainteté" dans la deuxième partie du livre: "Soyez saint car je suis saint». Cette sainteté, qui n'a pas encore un caractère rituel technique (Ibid.: 222), devient effective avec de telles règles de pureté établissant une frontière intangible entre les dévots de ce dieu à la haute sacralité et ceux qui y sont étrangers. 
laisse en effet voir de nombreux parallèles, le second consistant en l'élimination d'entités démoniques par, notamment, le sang du sacrifice et des incantations (Milgrom 2004 : 163-166). Or, ces démons, comme la Lamaštu coupable de bien des maux dont les fausses couches des femmes, étaient des êtres hybrides par excellence, pouvant être représentés sur des amulettes avec des animaux au statut ambigu ${ }^{36}$. C'est aussi dans ce contexte de représentations religieuses qu'il faut resituer la taxonomie biblique.

Paris

ch.lemardele@gmail.com

MOTS CLÉS/KEYWORDS: Mary Douglas - Bible - anthropologie des religions/anthropology of religion - Jacob Milgrom - philologie/philology - théologie/theology - Lévitique/Leviticus Deutéronome/Deuteronomy - taxonomie/taxonomy - système symbolique/symbolic system animaux purs et impurs/clean and unclean animals - tabou alimentairelfood taboo.

36. Sur une amulette mésopotamienne d'environ 800 av. J.-C., conservée au British Museum, on peut voir la Lamaštu à gueule de lion debout sur un cheval, l'agrippant avec ses serres d'aigle, tenant un serpent dans chaque main et nourrissant au sein un chien et un porc. 
Benkheira, Mohamed Hocine

2000 Islam et interdits alimentaires. Juguler l'animalité. Paris, Presses universitaires de France.

\section{Bonte, Pierre}

1999 «Sacrifices en islam : textes et contextes ", in Pierre Bonte, Anne-Marie Brisebarre \& Altan Gokalp, Sacrifices en islam. Espaces et temps d'un rituel. Paris, CNRS Éd. ("CNRS Anthropologie») : 21-61.

\section{Calvez, Marcel}

2006 «L'analyse culturelle de Mary Douglas : une contribution à la sociologie des institutions», Sociologies [En ligne: http://sociologies.revues.org/522].

De Heusch, Luc

2007 "Mary Douglas (1921-2007)", L'Homme 184 : 215-220

[http://lhomme.revues.org/14072].

\section{Detienne, Marcel}

2005 Les Grecs et nous. Une anthropologie comparée de la Grèce ancienne. Paris, Perrin (" Pour l'histoire»).

\section{Douglas, Mary}

1973 "Critique and Commentary", in Jacob Neusner, The Idea of Purity in Ancient Judaism. Leiden, E. J. Brill : 137-142.

1975 Implicit Meanings. Essays in Anthropology. London, Routledge \& Kegan Paul.

1978 Cultural Bias. London, Royal Anthropological Institute ( Royal Anthropological Institute Occasional Paper» 35).

1992 [1966] De la souillure. Études sur la notion de pollution et de tabou. Trad. de l'anglais par Anne Guérin. Paris, La Découverte ("Textes à l'appui. Série Anthropologie "). [Éd. orig. : Purity and Danger. An Analysis of Concepts of Pollution and Taboo, London, Routledge \& Kegan Paul, 1966.] 1993a "The Forbidden Animals in Leviticus ", Journal for the Study of the Old Testament $59:$ 3-23.

1993b In the Wilderness. The Doctrine of Defilement in the Book of Numbers. Sheffield, Sheffield Academic Press.

1996a [1970] Natural Symbols. Explorations in Cosmology. London, Routledge.

1996b "Sacred Contagion ", in John F. A. Sawyer, ed., Reading Leviticus. A Conversation with Mary Douglas. Sheffield, Sheffield Academic Press : 86-106.

1998 "La pureté du corps", Terrain 31 :

5-12 [http://terrain.revues.org/3131].

1999 [1986] Comment pensent les institutions. Suivi de La connaissance de soi et Il n'y a pas de don gratuit. Trad. révisée de l'anglais par Anne Abeillé. Paris, La Découverte ( Recherches. Série Bibliothèque du MAUSS").

2000 "Impurity of Land Animals ", Marcel J. H. Poorthuis \& Joshua Schwartz, eds, Purity and Holiness. The Heritage of Leviticus. Leiden, Brill : 33-45.

2002 "Le bouc qui s'en va ", Pardès 32-33: 129-134 [http://www.cairn.info/revuepardes-2002-1-page-129.htm].

2003 "The Go-Away Goat ", in Rolf Rendtorff, Robert A. Kluger, eds, The Book of Leviticus. Composition and Reception. Leiden-Boston, Brill : 121-141. 2004a [1999] L'Anthropologue et la Bible. Lecture du Lévitique. Trad. de l'anglais par Jean L'Hour. Paris, Bayard.

2004b Jacob's Tears. The Priestly Work of Reconciliation. Oxford, Oxford University Press.

2004c "Why I Have to Learn Hebrew: The Doctrine of Sanctification ", in Thomas Ryba, George Bond \& Herman Tull, eds, The Comity and Grace of Method. Essays in Honor of Edmund F. Perry. Evanston, Northwestern University Press : 147-165. 2007 «Pour ne plus entendre parler de la "culture traditionnelle" ", Revue du MAUSS 29: 479-516. 


\section{Faure, Laurence}

2010 «Sens et enjeux d'un interdit alimentaire dans le judaïsme : l'exemple de couples juifs ashkénazes à Londres ", Anthropology offood 7 [http://aof.revues.org/6548].

Hendel, Ronald Stephen

2008 «Remembering Mary Douglas :

"Kashrut", Culture, and Thought-Styles ", Jewish Studies 45 : 3-15.

\section{Houston, Walter}

1993 Purity and Monotheism. Clean and Unclean Animals in Biblical Law. Sheffield, JsOT Press (" Journal for the study of Old Testament. Supplement Series»140).

\section{Klawans, Jonathan}

2006 Purity, Sacrifice, and the Temple. Symbolism and Supersessionism in the Study of Ancient Judaism. Oxford-New York, Oxford University Press.

\section{Lassave, Pierre}

2011 L'Appel du texte. Sociologie du savoir bibliste. Rennes, Presses universitaires de Rennes ("Sciences des religions").

\section{Lemardelé, Christophe}

2002 «Le sacrifice de purification: un sacrifice ambigu?", Vetus Testamentum $52: 284-289$.

2007 «Mary Douglas, L’Anthropologue et la Bible : lecture du Lévitique", Revue de l'histoire des religions 224 : 111-114 [http://rhr.revues.org/5239].

2011-2012 «Le sacrifice juif dans le système sacrificiel antique : problèmes épistémologiques dans l'historiographie moderne ", Annuaire de l'École pratique des hautes études, Sciences religieuses 120 : 217-221 [http://asr.revues.org/1177].

\section{Lenclud, Gérard}

2000 [1991] "Douglas, Mary ", in Pierre Bonte \& Michel Izard, eds, Dictionnaire de l'ethnologie et de l'anthropologie. Paris, Presses universitaires de France : 203.
Levine, Baruch A.

1974 In the Presence of the Lord. A Study of Cult and Some Cultic Terms in Ancient

\section{Lévi-Strauss, Claude}

1962 La Pensée sauvage. Paris, Plon.

Marx, Alfred

2011 Lévitique 17-27. Genève, Labor \& Fides ( Commentaire de l'Ancien Testament »).

Milgrom, Jacob

1983 Studies in Cultic Theology and Terminology. Leiden, Brill ( Studies in Judaism in Late Antiquity» 36).

1991 Leviticus 1-16. A New Translation with Introduction and Commentary. New York, Doubleday.

1992 "Two Biblical Hebrew Priestly Terms : šeqes and tāmé'", Maarav 8 : 107-116.

2004 Leviticus. A Book of Ritual and Ethics. Minneapolis, Fortress Press.

\section{Nihan, Christophe}

2007 From Priestly Torah to Pentateuch. A Study in the Composition of the Book of Leviticus. Tübingen, Mohr Siebeck.

Schmidt, Francis

1994 La Pensée du Temple. De Jérusalem à Qoumrân. Identité et lien social dans le judä̈sme ancien. Paris, Le Seuil (« La Librairie du XXe siècle »).

\section{Schmitt, Rüdiger}

2008 "The Problem of Magic and Monotheism in the Book of Leviticus", Journal of Hebrew Scriptures 8 : 2-12 [http://www.jhsonline.org/Articles/ article_88.pdf].

\section{Uehlinger, Christoph}

2004 "Genèse 1-11 ", in Thomas Römer, Jean-Daniel Macchi \& Christophe Nihan, eds, Introduction à l'Ancien Testament. Genève, Labor \& Fides ( Le Monde de la Bible») : 114-133. 
Christophe Lemardelé, Mary Douglas et la Bible: la (re)conversion d'une anthropologue.De Purity and Danger dans les années 1960 à Leviticus as Literature trente ans plus tard, le parcours de l'anthropologue britannique Mary Douglas peut sembler être le simple passage d'un intérêt quelque peu "exotique " pour la Bible à un véritable intérêt intellectuel et scientifique. En effet, après avoir choisi "Les abominations du Lévitique" comme exemple à sa démonstration sur les notions de pureté et d'impureté, l'anthropologue en venait à étudier l'exemple pour luimême. Mais à y regarder de plus près, on peut penser aussi qu'il ne s'est pas seulement agi d'une évolution plus ou moins limpide, on peut même penser qu'il y a une rupture dans le parcours. Non seulement Mary Douglas a cessé d'être anthropologue en devenant bibliste, mais elle est totalement revenue sur ses premiers pas pour en effacer les traces et suivre celles d'un rabbin philologue au pas très assuré: Jacob Milgrom. Pourtant, malgré l'hétérogénéité des facteurs contextuels d'explication, la clé d'interprétation énoncée par Douglas en 1966 garde toute sa pertinence.
Christophe Lemardelé, Mary Douglas and the Bible:The (re)Conversion of an Anthropologist.From Purity and Danger in the 1960's to Leviticus as Literature thirty years later, Mary Douglas's itinerary seemingly passes from a somewhat "exotic" to a genuine intellectual and scientific interest in the Bible. After having chosen "The Abominations of Leviticus" to demonstrate her concepts of purity and impurity, this British anthropologist studied this example for its own sake. A closer look leads us to think that this was not just a more or less clear development, but that it marked a rupture in her itinerary. Mary Douglas not only stopped being an anthropologist when studying the Bible but also came back on her beginnings to erase her itinerary and follow the track firmly imprinted by Jacob Milgrom, a rabbi and philologist. Despite the variety of contextual explanatory factors, the key of interpretation formulated by Douglas in 1966 is still fully pertinent. 